Brendan Smyth, Ceridwen Jones and John Saunders, the authors of the article, comment:

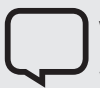
We thank Dr Richards for his comments which highlight the growing interest in extending the use of metformin to patients with moderate to severe chronic kidney disease. Within the constraints of our brief review we preferred to adhere to a conservative recommendation regarding metformin use in end-stage renal disease without describing the current controversy. We agree that recent studies have substantially altered our understanding of the risk-benefit profile of metformin in patients with chronic kidney disease and we look forward to larger studies with substantial follow-up of safety end points. However, until these are available we would continue to suggest that metformin not be used in dialysis patients except under the close supervision of a nephrologist or as part of a clinical trial.

\section{Anal fissures and liquid paraffin}

Aust Prescr 2016:39:75

http://dx.doi.org/10.18773/austprescr.2016.040

Congratulations on the concise article on anal fissure.' It covered pathology and management well with one exception, the role of stool softening.

While the cause of an anal fissure is unknown, the condition is perpetuated by the cycle:

pain $\rightarrow$ internal sphincter spasm $\rightarrow$ faecal retention $\rightarrow$ hard stool $\rightarrow$ rapid anal stretching at (inevitable) defaecation $\rightarrow$ opening of the fissure $\rightarrow$ pain

This cycle can be broken by adequate stool softening. Increasing fibre intake is slow to act and often fails to give adequate softening. The stool needs to be as soft as toothpaste.

Osmotic agents (e.g. macrogol) or non-absorbed sugars (e.g. lactulose) can be used but have their disadvantages. The cheapest, most rapid acting product is liquid paraffin. A large dose can be given initially so that within 24 hours defaecation is pain free or almost so. In children, depending on their age, a dose of 20-30 mL to start can be followed by $10-15 \mathrm{~mL}$ twice daily. The dose can be easily adjusted according to the response. Liquid paraffin can be continued for two to three months, after which it is reduced slowly. Fibre can then be used if hard stools are a long-term problem.
As paraffin is not digested, does not change intestinal movement, and does not cause movement of water in or out of the gut, it is relatively safe. Some leaking of oil is seen occasionally but this can be controlled by lowering the dose by a few $\mathrm{mL}$.

I have yet to find good evidence of a complication other than pulmonary aspiration secondary to gastro-oesophageal reflux. It is best to avoid giving a dose last thing at night. Macrophages in intestinal nodes can show micro droplets but no ill effect has been noted. About 30 years ago there was a belief that liquid paraffin could cause fat soluble vitamin deficiency, however this has not been substantiated by subsequent research. ${ }^{2}$

\section{Hugh Martin}

Paediatric surgeon

Sydney

\section{REFERENCE}

1. Schlichtemeier S, Engel A. Anal fissure. Aust Prescr 2016:39:14-7. http://dx.doi.org/10.18773/ austprescr.2016.007

2. Sharif $F$, Crushell E, O'Driscoll K, Bourke B. Liquid paraffin: a reappraisal of its role in the treatment of constipation. Arch Dis Child 2001;85:121-4. http://dx.doi.org/10.1136/ adc.85.2.121

Steven Schlichtemeier and Alexander Engel, the authors of the article, comment:

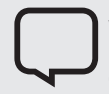

We thank Dr Martin for his response detailing the use of liquid paraffin as part of the conservative management of patients with acute anal fissures. We agree that increased fibre intake may not provide adequate stool softening and often an additional or alternate agent may be necessary to achieve adequate stool consistency. Its effectiveness, cost and low adverse-effect profile may make liquid paraffin the product of choice in this situation. It may even be the preferred agent to begin with in children as a small amount of liquid may be favoured over spoonfuls of fibre.

Unfortunately, most studies combine increased fibre and laxatives with sitz baths as part of best supportive management in comparison to topical treatment, botulinum injections or surgery. There is no good-quality evidence comparing increased fibre to the different classes of laxatives in the management of anal fissures. However, stool softeners may benefit the patient without the need for a drug, injection or surgery. 